\title{
Journal of Immunology and Allergy
}

An Open Access Journal

\section{Amelioration for Allergic Rhinitis and Asthma: The Treated Yeast Milmed and Physical Exercise}

\author{
Trevor Archer ${ }^{1,2^{*}}$,Rose Mary Erixon ${ }^{1,2}$, Thomas Lenz ${ }^{1}$ and Rita Businaro ${ }^{3}$ \\ ${ }^{1}$ Milmed Unico AB, Regeringsgatan 93, Stockholm, Sweden. \\ ${ }^{2}$ University of Gothenburg, Department of Psychology, Gothenburg, Sweden.
}

${ }^{3}$ Sapienza University of Rome, Department of Medico-Surgical Sciences and Bio techniques, Rome, Italy.

*Corresponding Author: Archer Trevor, Milmed Unico AB, Regeringsgatan 93, Stockholm, Sweden.

Received Date:10-28-2019; Accepted Date:11-04-2019; Published Date:11-19-2019

Copyright $^{\odot} 2019$ by Archer T, et al. All rights reserved. This is an open access article distributed under the terms of the Creative Commons Attribution License, which permits unrestricted use, distribution, and reproduction in any medium, provided the original author and source are credited.

\section{Keywords}

Allergic Rhinitis; Asthama; Yeast Milmed; Physical Excercise

\section{Introduction}

In the developed, industrial world, about $20 \%$ of people are affected by allergic rhinitis, about $6 \%$ of people have at least one food allergy, and about $20 \%$ have atopic dermatitis at some point in time. The global prevalence of allergies and asthma has increased, quite exponentially, during the last decades with WHO estimating above 400 million individuals debilitated by allergic rhinitis and 300 million by asthma; furthermore, 25-32\% of European populations remain affected [1,2]. The postulated links between these conditions [3] demand a mandatory integration between to optimize treatment effects $[2,4]$. In some countries, up to $18 \%$ of people have asthma. Rates of many allergic diseases appear to be increasing. More than 150 million Europeans suffer from chronic allergic diseases and the current prediction is that by 2025 half of the entire EU population will be affected (EAACI, 2016).

An allergy presents a chronic condition involving an abnormal reaction to an ordinarily harmless substance called an allergen. Allergens may include aero-allergens such as dust mite, mold, tree weed and grass pollen, as well as food allergens such as milk, egg, soy, wheat, nut or fish proteins. Symptoms of allergy may include red eyes, an itchy rash, itching in the nose, roof of the mouth, throat, eyes, sneezing, a stuffy nose (congestion), a runny nose, tearing eyes, shortness of breath, or swelling. Food allergies may present with vomiting, diarrhea, respiratory symptoms or anaphylaxis immediately after ingestion of the allergen.

These diseases include hay fever (allergic rhinitis), asthma, allergic eyes (allergic conjunctivitis), allergic eczema, hives (urticaria), and allergic shock (also called anaphylaxis 
and anaphylactic shock). Hay fever (allergic rhinitis) is the most common of the allergic diseases and refers to seasonal nasal symptoms that are due to pollens. Year round or perennial allergic rhinitis is usually due to indoor allergens, such as dust mites or molds. The most common symptoms include runny nose, stuffy nose, sneezing, nasal itching (rubbing), itchy ears and throat and postnasal drip (throat clearing).

Asthma presents a chronic breathing problem those results from the allergic-inflammation and spasm of the lung's air passages (bronchial tubes). Asthma is most often, but not always, related to allergies. Common symptoms include shortness of breath, wheezing, coughing and chest tightness.

Allergic eyes (allergic conjunctivitis) is inflammation of the tissue layers (membranes) that cover the surface of the eyeball and the undersurface of the eyelid. The inflammation occurs a result of an allergic reaction. Common symptoms are redness under the lids and of the eye overall, watery, itchy eyes and swelling of the membranes.

Allergic eczema is an allergic rash that is usually not caused by skin contact with an allergen and features the following symptoms itching, redness, and or dryness of the skin, rash on the face, especially children and rash around the eyes, in the elbow creases, and behind the knees, especially in adults.

Hives (urticarial) are skin reactions that appear as itchy swellings and can occur on any part of the body. Hives can be caused by an allergic reaction, such as to a food or medication, but they also may occur in non-allergic people. Typical hive symptoms are raised red welts and intense itching.

Treatments for allergies include avoiding known allergens and the use of medications such as antihistamines and steroids. These may be in the form of pills or liquid, nasal sprays, or eyedrops. Although newer generation of antihistamines has improved, people may still experience side effects, such as headache, tiredness, dizziness, dry mouth, vision changes and excitability/nervousness. Side effects of corticosteroid nasal sprays include nosebleeds, stinging in the nose and dryness of the nose, nausea and dizziness.

In addition, several complementary health approaches have been studied for allergic rhinitis and there is some evidence that a few may be helpful. Probiotics (live microorganisms that may have health benefits) have been investigated for diseases of the immune system, including allergies. For example, JP 6002452 discloses a Saccharomyces cerevisiae yeast as useful for prevention and treatment of various immunological diseases by suppressing production of IgE causing type I allergy symptoms and JP 4712289 discloses a mixture of Saccharomyces and lactic acid bacteria for the prevention of food allergy. Although some studies have had promising results, the overall evidence on probiotics and allergic rhinitis is inconsistent.

Current treatments for allergies, applying, for example antihistamines, decongestants, lotions and creams and steroids, bronchodilators or glucocorticoids [5] may be found wanting over 
two main avenues: (a) therapeutic-limitation and (b) necessary avoidance of specificunspecific allergens. Thus, there exists an ever-increasing need for new chemical compositions/entities and methods that allow for improved interventions for the alleviation of allergy and/or symptoms associated with allergy.

The purpose of the present treatise is to describe novel interventions that may offer alternative means to ameliorate these problems that pertain, essentially, to environmental provocation to a compromised immune system.

\section{Anti-allergic effects of Milmed}

The millimeter-wavelength treated yeast, that has been allocated the name, Milmed, with anti-neurodegenerative properties [6], was applied recently for the treatment of recurrent seasonal and animal hair allergies over eight-week twice weekly administrations. The effects were found to be markedly effective in ameliorating the discomfort and problems of the twenty-two individuals who had applied to participate in the study. Limited details and outcomes for nine of these are described below: -

Patient 1. Female, 42 years old. Non-smoker. History of allergy: pollen, grass, mites, dog and cat. Tiredness, frequent colds. Nasal obstruction, sneezing, reduced ability to smell, itchy, watery and swollen eyes; heavy breathing, shortness of breath, wheezing breath, thick mucus, and chest tightness. Intake of Milmed every morning before breakfast for Y weeks. Perceived improvement of allergic problems on scale of 1 to 10 rated as 8 out of 10 ; reduced need to take allergy medicine; is less tired.

Patient 3. Male, 27 years old. Non-smoker but snuff-user. History of allergy: pollen/grassseasonal-April to August inclusive, symptoms: runny-blocked nose, itchiness/pruritus, tears, and redness. No childhood indications, onset 2 years previously, no treatments used. Nongenetic. Intake of Milmed from start before breakfast. Perceived improvement on scale of 1 to 10 rated as 10; no parallel medication; mild cold.

Patient 4. Female, 88 years old. Non-smoker. No childhood indications, onset 57 years previously. History of allergy: fur, cats and dogs, contact. Throughout the year. Throat constriction, hoarseness, sneezing. Use of a variety of prescribed medications against fur allergy. Non-genetic. Intake of Milmed from start before breakfast. Perceived improvement on scale of 1 to 10 rated as 10; no colds since Milmed, highly pleased with Milmed effect.

Patient 5. Male, 42 years old. Non-smoker. No childhood indications. History of allergy: pollen/grass-seasonal-April to August inclusive, symptoms: runny-blocked nose, itchiness/pruritus, onset 11 years previously. Medication: Clarityn, nasal spray, seasonal May, June, July. Non-genetic, medication above. Intake of Milmed from start before breakfast. Perceived improvement on scale of 1 to 10 rated as 10 ; no colds or illness, since Milmed.

Patient 6. Male, 49 years old. Non-smoker but snuff. No childhood indications, onset 10 years previously. History of allergy: pollen/grass-seasonal-May to August inclusive, 
symptoms: runny-blocked nose, swelling, medication: Clarityn, nasal spray. Non-genetic. Intake of Milmed from start before breakfast. Perceived improvement on scale of 1 to 10 rated as 10; no colds or illness, since Milmed.

Patient 9. Male, 71 years old. Non-smoker. No childhood indications. Onset 48 years previously. History of allergy: horse, pollen-seasonal-April to June inclusive, symptoms: runny-blocked nose, swelling, itchiness in eyes. Medication: use of a variety medication against allergy. Non-genetic. Intake of Milmed from start before breakfast for X weeks.Perceived improvement on scale of 1 to 10 rated as 10 out of 10 ; no parallel medications; more alert since Milmed.

Patient 12. Female, 46 years old. Non-smoker. Childhood indications: Eczema, asthma/ bronchial problems, allergic rhinitis, food hypersensitivity. History of allergy: pollen, grass, dust, mites, dog, cat, horse, timothy, hazelnut, almond, apple, kiwi, peach. Throughout the year; symptoms: blocked nose, swelling, sneezing, itchiness, redness and swollen eyes; throat constrictions, cough, hoarseness, heavy breathing, shortness of breath, wheezing breath, thick mucus, pain in the chest. Medication: Pulmicort, bricanyl, omeprazole, betaproc. Genetic. Intake of Milmed from start before breakfast for X weeks. Perceived improvement on scale of 1 to 10 rated as 8 out of 10 ; reduced need to take allergy medicine; more alert; better health, less illness.

Patient 14. Male, 48 years old non-smoker. Childhood indications: allergic rhinitis, food hypersensitivity. History of allergy: pollen, grass, dust, fur, dog, cat, horse, birch, apple, scents-seasonal-March to October inclusive, symptoms: blocked and swelling nose, sneezing, itchiness and watery eyes; itchiness, irritation and swollen oral cavity / pharynx; throat constrictions, cough, hoarseness, heavy breathing. Medication: Loratadine. Non genetic. Intake of Milmed from start before breakfast for X weeks. Perceived improvement on scale of 1 to 10 rated as 6 out of 10; reduced need to take allergy medicine.

Patient 16. Male, 34 years old. Non-smoker but snuff. Childhood indications: allergic rhinitis, onset 20 years previous. Medication; Loratadine and mommox. History of allergy: pollen, grass, dust, fur, dog, cat, horse, seasonal-April to September, symptoms: blocked and swelling nose, sneezing; itchiness, watery and redness eyes. Non genetic. Intake of Milmed from start before breakfast for 8 weeks, missed 1 week [NB. Could have affected result]. Perceived improvement on scale of 1 to 10 rated as 7 out of 10 ; no parallel allergy medication, Milmed has replaced the previous allergy medicine, highly pleased with Milmed effect.

\section{Anti-allergic effects of exercise}

Regular, long-term physical exercise (at least 3-4 days per week over years rather than months) promotes a series of reactions, responses, adaptive behaviors and physiological adaptations (cf. hormesis) that are intimately dependent upon different aspects of the exercise-activity schedules, such as duration, volume, and intensity and whether or not it is endurance or resistance. In this context, the relative discrepancies associated with mild as 
opposed to intensive whereby the former would promote ameliorative effects and the latter worsened reactions, ought to be observed [6]. The positive interventional influences of physical exercise for dispositions of poor health, disorder and disease over a wide range of immunologically dependent parameter has been discussed comprehensively [7]. Suffice it to say that the influence of exercise among individuals suffering (i) acutely, and (ii) chronically, from allergic reactions may vary considerably. Physical exercise, despite presiding propensity to precipitate reactions depending on acute vs. chronic parameters, in the various forms of swimming, treadmill-running, aerobic or resistance, has been recommended for individuals prone to allergic rhinitis and/or asthma $[8,9,10]$.In a longitudinal, randomized-control intervention study examining the potentially therapeutic effects of 'recreation winter exercise' upon a group of individuals presenting allergic airway inflammation expressing allergic rhinitis and asthma, estimations of quality-of-life, spirometry and cardiovascular fitness following a ten-day winter sports exercise program were derived. There were significant improvements in fractional oral exhaled nitric oxide accompanied by a significant decrease iron nitrate, and improvements in nasal eosinophilic cell counts due to exercise that prompted the conclusion of sustainable improvements in allergic symptoms. It has been demonstrated repeatedly that physical exercise combined with Milmed treatment over six to fourteen weeks induced a synergistic effect against symptoms of dopamine neurodegeneration [11,12]. The present account promotes the contention the physical exercise with accompanying Milmed treatment may offer a novel and viable alternative to anti-allergy interventions under current usage.

\section{Conclusion}

Treated yeast, Milmed, by itself or in combination with physical exercise programs may offer a novel viable alternative to current therapeutic alternatives available for ameliorating the problems and discomforts occasioned by allergens.

\section{References}

1. Biedermann T, Winther L, Till SJ, Panzner P, Knulst A, Valovirta E Birch pollen allergy in Europe. Allergy. 2019;74(7):1237-48.

2. Bousquet J, Bedbrook A, Czarlewski W, Onorato GL, Arnavielhe S, Laune D et al The MASK study group Guidance to 2018 good practice: ARIA digitally-enabled, integrated, person-centered care for rhinitis and asthma. Clin Transl Allergy. 2019;9:16.

3. Shaaban R, Zureik M, Soussan D, Neukirch C, Heinrich J, Sunyer J, et al. Rhinitis and onset of asthma: a longitudinal population-based study. Lancet. 2008;372(9643):1049-57.

4. Bousquet J, Schünemann HJ, Togias A, Bachert C, Erhola M, Hellings PW et al. Allergic Rhinitis and Its Impact on Asthma Working Group Next-generation Allergic Rhinitis and Its Impact on Asthma (ARIA) guidelines for allergic rhinitis based on Grading of Recommendations Assessment, Development and Evaluation (GRADE) and real-world evidence. J Allergy Clin Immunol. Pii.2019S0091-6749(19)31187

5. Vandenplas $O$, Vinnikov D, Blanc PD, Agache I, Bachert C, Bewick M, et al. Impact of Rhinitis on Work Productivity: A Systematic Review. J Allergy Clin Immunol Pract. 2018 6(4):1274-86. 
6. Terra R, Alves PJF, Lima AKC, Gomes SMR, Rodrigues LS, Salerno VP, Da-Silva SAG, Dutra PML Immunomodulation from Moderate Exercise Promotes Control of Experimental Cutaneous Leishmaniasis. Front Cell Infect Microbiol. 2019;9:115.

7. Dua S, Ruiz-Garcia M, Bond S, Durham SR, Kimber I, Mills $C$ et al. Effect of sleep deprivation and exercise on reaction threshold in adults with peanut allergy: A randomized controlled study. Allergy Clin Immunol. 2019;15. pii: S0091-6749(19)30934-0.

8. Avallone KM, McLeish AC Asthma and aerobic exercise: a review of the empirical literature. $J$ Asthma. 2013; 50(2):109-16.

9. Carson KV, Chandratilleke MG, Picot J, Brinn MP, Esterman AJ, Smith BJ Physical training for asthma. Cochrane Database Syst Rev. 2013;9

10. Silva D, Moreira A The role of sports and exercise in allergic disease: drawbacks and benefits. Expert Rev Clin Immunol. 2015;11(9):993-1003.

11. Archer T, Fredriksson A The yeast product Milmed enhances the effect of physical exercise on motor performance and dopamine neurochemistry recovery in MPTP-lesioned mice. Neurotoxicity Research. 2019;24, 393-406.

12. Archer T, Garcia D, Fredriksson A Restoration of MPTP-induced deficits by exercise and Milmed $®$ co-treatment. Peer J. 2014

13. Giavina-Bianchi P, Aun MV, Takejima P, Kalil J, Agondi RC United airway disease: current perspectives. J Asthma Allergy. 2016;9:93-100. 